\title{
Joint adaptive beamforming and perfect power control in wireless networks over power control error
}

\author{
Mohamad Dosaranian-Moghadam \\ Department of Electrical Engineering, Qazvin Branch, Islamic Azad University, Qazvin, Iran. \\ m_dmoghadam@qiau.ac.ir
}

\begin{abstract}
In this paper, we use joint perfect power control (PPC) and adaptive beamforming algorithms as constrained least mean square (CLMS) and conjugate gradient adaptive beamforming (CGBF) in a 2D urban environment. The CLMS algorithm is capable of efficiently adapting according to the environment and able to permanently maintain the chosen frequency response in the look direction while minimizing the output power of the array. In addition, with the CGBF algorithm, the desired users' signal in an arbitrary path is passed and the inter-path interference (IPI) is canceled in other paths in each RAKE finger. The adaptability of the algorithms is closely observed for uniformly spaced linear array. In addition, in this paper, we present switched-beam (SB) technique. In the SB technique by using a number of fixed, independent, or directional antennas we increase the uplink capacity of the wireless systems. Also, we study an analytical approach for the evaluation of the impact of power control error (PCE) on wireless communication systems. Simulation results indicate that the SB technique is able to considerably increase the signal to interference plus noise ratio (SINR) of desired user in comparison with other algorithms. In addition, we observe that the SINR in the CLMS algorithm is lower than the CGBF algorithm. Finally, we discuss two parameters of the PCE and path-loss exponent and their effects on capacity of the system via some computer.
\end{abstract}

Keywords: Adaptive beamforming, Power control, Wireless networks.

\section{Introduction}

It is generally acknowledged that a viable approach for increasing the capacity of wireless cellular systems in the reverse link (uplink) is represented by antenna arrays (Chang et al., 2002; Abrardo, 2003; Wang, 2009; Kumatani et al., 2009; Zhang et al., 2009; Yang \& Fang, 2009). There are two main techniques that are used to exploit transmit antenna arrays. The first one is space-time coding, which provides diversity in fading environments (Cai \& de Lamare, 2009). The second is beamforming, which provides spatially matched transmission or (in multiuser scenarios) mitigates interference (Abdel-Samad et al., 2009; Nai et al., 2009; Wang \& Payaro, 2010; El-Keyi \& Champagne, 2010). The power gain that is achieved by transmit beamforming is proportional to the number of transmit antennas. The goal of adaptive beamformers is to enhance the desired signal and suppress noise as well as strong interferences at the sensor array output simultaneously. In past years, some interesting approaches for adaptive beamforming have been suggested to provide robustness against various mismatches in the channel state information for line of sight (LoS) propagation environments (Lorenz \& Boyd, 2005; El-Keyi \& Champagne, 2010; Lawrence, 2010). In addition, the problem of robust beamforming for flat fading channels was considered by Abdel-Samad et al. (2006). Also Park et al. (2009) proposed a new beamforming scheme which can transmit multiple data streams simultaneously in multiple-input multiple-output (MIMO) channels by generalizing transmit maximal ratio combining technique. Recently, the robustness of transmit beamforming from the worst-case robustness perspective is studied by Wang (2010). In this paper, we use three adaptive beamforming methods for wireless systems in 2D urban environments for reverse link. For this purpose and in order to realize a 2D urban environment, urban signal propagation simulator (USPS) is designed. This software is implemented for both forward link and reverse link (Akhlaghi et al., 2004; Dosaranian-Moghadam et al., 2009; Dosaranian-Moghadam et al., 2010; Dosaranian-Moghadam et al., 2010; Dosaranian-Moghadam et al., 2012).

It is well known that in order to fully exploit the potential advantage of cellular systems, power control is required to counteract the effects of the near-far problem. The wireless system capacity is maximized if each mobile transmitter power level is controlled so that its signal arrives at the base station (BS) with the minimum required signal to interference plus noise ratio (SINR) (Ye \& Haimovich, 2000; Abrardo \& Sennati, 2000; Wang \& Yu, 2001; Rintamaki et al., 2004; Qian \& Gajic, 2006; Carrasco \& Femenias, 2008). However, when applying power control in practice the performance is restricted by a number of limitations and therefore, perfect power control cannot be achieved. The issue of the effect of power control errors on wireless systems has received a great deal of attention over the last few years (Ye \& Haimovich, 2000; Abrardo \& Sennati, 2000; Carrasco \& Femenias, 2008). In this paper, we consider the PPC and the effect of power control error (PCE) on wireless cellular systems. 


\subsection{Propagation model in 2D urban environment}

Because of using 2D urban structure in this paper, for computing yield for the path between a mobile set (MS) and base station (reverse link), propagation model is dramatized in urban environments. Accordingly, with USPS software, mobile set antenna radiate beams which diffuse in all directions and parts of the beams reach to base station (BS). In this software, delivered beam from mobile set by the time of collision to an obstacle like a wall surface or a building, reflects to a new angle and continues its path, which is called reflection phenomena. In condition that radiated beam is conflicted to an obstacle edge, then diffraction phenomena is happened and diffracting point is diffusing new beams to all directions like a transmitter. All reflected beams will stay in the environment as long as their power is not reduced to a threshold limit. Fig. 1 shows both phenomena for LoS and non-LoS paths in reverse link. On the other hand, in this software, the channel is modeled with lognormal distributed shadowing. Accordingly, every pixel of the environment stores all environmental information like receiving power and angle for each path (Akhlaghi et al., 2004; Dosaranian-Moghadam et al., 2009; Dosaranian-Moghadam et al., 2010; Dosaranian-Moghadam et al., 2010; Dosaranian-Moghadam et al., 2012).

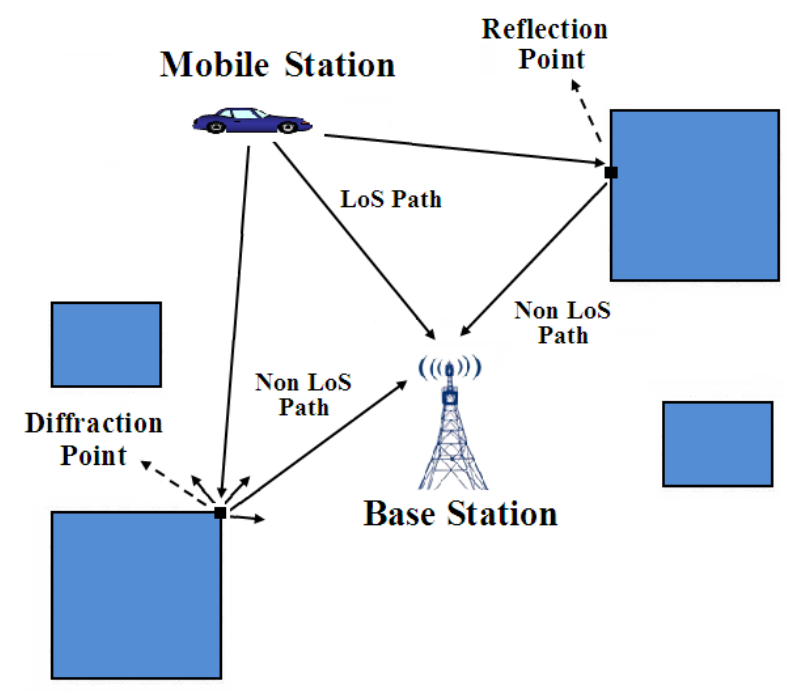

Fig.1. Diffraction phenomena and reflection phenomena in the USPS software (LoS and Non-LoS paths) for a 2D urban environment in reverse link.

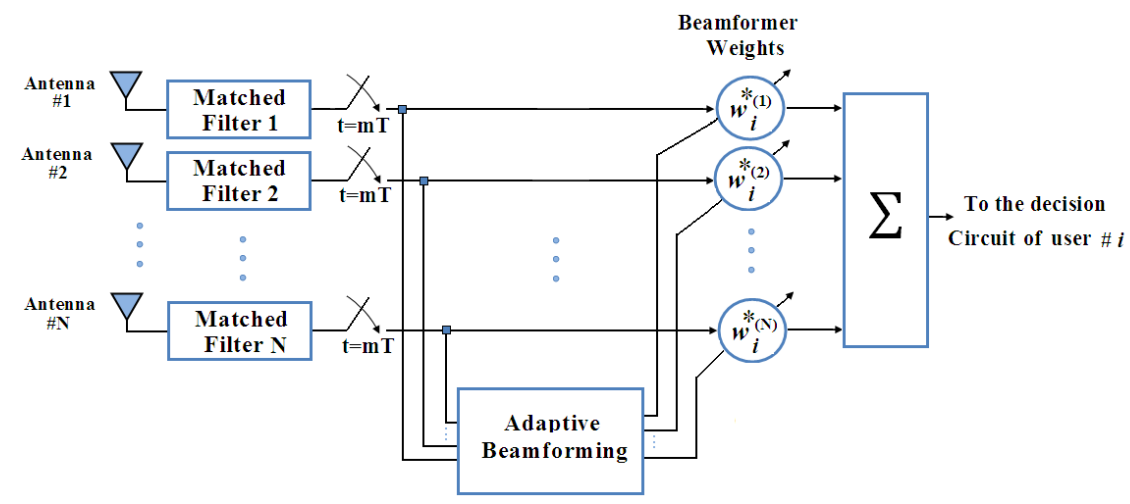

Fig.2. Antenna array and beamformer (Shakir \& Durrani, 2007)

According to the above dramatization, we could see that because of LoS in un-urban environment, only one signal is delivered from each user to a receiver, while in function and because of the elimination phenomena in urban environments, beside to signals which are delivered to line sight, signals which have difference in phase or domain with this signal are also received by the receiver.

\subsection{System model}

An adaptive antenna array consists of a set of antennas, designed to receive signals radiating from some specific directions and attenuate signals radiating from other directions of no interest. The outputs of array elements are weighted and added by a beamformer, as shown in Fig. 2, to produce a directed main beam and adjustable nulls. In order to reject the interference, the beamformer has to place its nulls in the directions of sources of interference, and steer to the direction of the target signal by maintaining constant 
gain at this direction (Rashid-Farrokhi et al., 1998; Shakir \& Durrani, 2007). It should be mentioned that the Doppler Effect and the effect of measurement errors have not been considered in the paper. Accordingly, in this paper, we focus on the uplink communication path in a single-cell wireless system with $M$ users and only one BS. In addition, we use a uniform linear array (ULA) of $N$ antenna elements in the base station.

Accordingly, the received signal vector in BS can be written as (Rashid-Farrokhi et al., 1998; Dosaranian-Moghadam et al., 2010; Dosaranian-Moghadam et al., 2012):

$$
\mathbf{x}(t)=\sum_{i=1}^{M} \sqrt{p_{i}^{\prime}} s_{i}\left(t-\tau_{i}\right) \mathbf{v}_{i}(\theta)+\mathbf{n}(t)
$$

where $s_{i}(t)$ is the message signal transmitted from the $i$ th user (desired user); $p_{i}^{\prime}$ is the received power of the $i$ th user in BS which is equal to $p_{i} G_{i}$ where $p_{i}$ and $G_{i}$ denote the transmitted power of the $I$ th user, and the link gain between user $i$ and BS in LoS path (or the best non-LoS path if LoS path is not exist), as shown in Fig. 1, respectively. It should be mentioned that for perfect power control (PPC), the received power is $p_{i}^{\prime}=P^{\prime}=E_{b} / T$ for all users connected to similar base station, where $T$ and $E_{b}$ are bit period and the energy per bit for all users, respectively. Also, $\mathbf{n}(t)$ is the thermal noise vector at the input of antenna array at BS and $\tau_{i}$ is the corresponding time delay. For simplicity, we further assume that the time delays for all uses are equal to 0 . Also the $N \times 1$ vector $\mathbf{v}_{i}(\theta)$ is the array response for user $i$ and can be written as follows (Rashid-Farrokhi et al., 1998).

$$
\mathbf{v}_{i}(\theta)=\sum_{l=1}^{L_{i}} \sqrt{\alpha_{i, l}} \mathbf{a}_{i}\left(\theta_{i, l}\right)
$$

where $L_{i}$ is the number of paths between user $i$ and BS in USPS; $\theta_{i, l}$ is the direction of arrival (DoA) in the $l$ th path for user $i ; \alpha_{i, l}$ is the normalized attenuation by the best link gain $\left(G_{i}\right)$ in USPS between user $i$ and BS in the $l$ th path, therefore $0<\alpha_{i, l} \leq 1$. Also, response of the base station array to the direction $\theta_{i, l}$ is given by

$$
\mathbf{a}_{i}\left(\theta_{i, l}\right)=\left[1 \exp \left(-j \tau_{\theta_{i, l}}\right) \ldots \exp \left(-j(N-1) \tau_{\theta_{i, l}}\right)\right]^{T}
$$

where $j=\sqrt{-1}$ and $\tau_{\theta_{i, l}}=(2 \pi d / \lambda) \sin \theta_{i, l}$, in which $\lambda$ is signal wavelength and $d$ is the distance between the antenna elements that for avoid the spatial aliasing should be defined as $d=0.5 \lambda$.

In non-spread spectrum systems, the transmitted signal is given by (Rashid-Farrokhi et al., 1998):

$$
s_{i}(t)=\sum_{m} b_{i}(m) g(t-m T)
$$

where $b_{i}(m)$ is the $i$ th user information bit stream with a bit period of $T$ and $g(t)$ is the pulse-shaping filter impulse response. It has been shown that the output of a matched filter sampled at the symbol intervals is a sufficient statistic for the estimation of the transmitted signal. The matched filter is sampled at $t=m T$

$$
\mathbf{x}(m)=\left.\mathbf{x}(t) * g^{*}(-t)\right|_{t=m T}
$$

Hence, the received signal at the output of the matched filter is given by

$$
\begin{aligned}
\mathbf{x}(m) & =\sqrt{P^{\prime}} b_{i}(m) \mathbf{v}_{i}(\theta)+\sum_{\substack{k=1 \\
k \neq i}}^{M} \sqrt{P^{\prime}} b_{k}(m) \mathbf{v}_{k}(\theta)+\mathbf{n}(m) \\
& =\sqrt{P^{\prime}} b_{i}(m) \mathbf{v}_{i}(\theta)+\mathbf{I}_{i}(m)+\mathbf{n}(m)
\end{aligned}
$$

where $\mathbf{I}_{i}(m)$ is the interference for user $i$ in BS and $\mathbf{n}(m)=\left.\mathbf{n}(t) * g^{*}(-t)\right|_{t=m T}$ (Dosaranian-Moghadam et al., 2010).

\subsection{The types of adaptive beamforming algorithms}

\subsubsection{Constrained LMS Algorithm}

Constrained LMS algorithm is a gradient based algorithm to minimize the total processor output power, based on the look direction constraint. The adaptive algorithm is designed to adapt efficiently in agreement with the environment and able to perma- 
nently preserve the desired frequency response in the look direction while minimizing the output power of the array. The combined form of the constraint is called constraint for narrowband beamforming (Rashid-Farrokhi et al., 1998; Xinyu et al., 2008; Shakir \& Durrani, 2007).

This form consider a narrowband beamformer where the narrowband signal from each element of smart antenna are multiplied by the complex weight calculated by using narrowband adaptive beamforming algorithm, and then summed to produce the output of the array. The definition of the complex weights of this beamformer in the $m$ th iteration for the $i$ th user is as follows (Litva \& Kwok-Yeung, 1996; Shakir \& Durrani, 2007; Xinyu et al., 2008).

$$
\mathbf{w}_{i}(m)=\left[w_{i}^{(1)}(m) w_{i}^{(2)}(m) \ldots w_{i}^{(N)}(m)\right]^{T}
$$

Accordingly, the output of the array in the $m$ th iteration for user $i$ is given by

$$
y_{i}(m)=\mathbf{w}_{i}^{H}(m) \mathbf{x}(m)
$$

The expected output power of the array in the $m$ th iteration is given by

$$
\begin{aligned}
\mathrm{E}\left(\left|y_{i}(m)\right|^{2}\right) & =\mathrm{E}\left(y_{i}(m) y_{i}^{*}(m)\right) \\
& =\mathrm{E}\left(\mathbf{w}_{i}^{H}(m) \mathbf{x}(m) \mathbf{x}^{H}(m) \mathbf{w}_{i}(m)\right)=\mathbf{w}_{i}^{H}(m) \mathbf{R}_{\boldsymbol{x}} \mathbf{w}_{i}(m)
\end{aligned}
$$

where $\mathrm{E}($.$) is denoted the expectation and \mathbf{R}_{\mathrm{ax}}$ is the correlation matrix of the received signal vector $\mathbf{x}(m)$.

A real-time CLMS algorithm for determining the optimal weight vector for user $i$ is (Shakir \& Durrani, 2007; Xinyu et al., 2008):

$$
\left\{\begin{array}{l}
\mathbf{w}_{i}(m+1)=\mathbf{w}_{i}(m)+\mu g\left(\mathbf{w}_{i}(m)\right) \\
\mathbf{w}_{i}^{H} \mathbf{a}_{i}\left(\theta_{i, \mathrm{~g}_{\max }}\right)=1
\end{array}\right.
$$

where $\mathbf{a}_{i}\left(\theta_{i, \mathrm{~g}_{\max }}\right)$ denotes spatial response of the array for user $i$ in the best path gain, i.e., $\alpha_{i, l}=1$, and $\mathbf{w}_{i}(m+1)$ is the new weight computed at the $(m+1)$ th iteration for user $i$. Also, the variable scalar $\mu$ denotes a positive scalar (gradient step size) that controls the convergence characteristic of the algorithm, that is, how fast and how close the estimated weights approach the optimal weights, and $g\left(\mathbf{w}_{i}(m)\right)$ denotes an unbiased estimate of the gradient of the power surface $\left(\mathbf{w}_{i}(m)^{H} \mathbf{R}_{x} \mathbf{w}_{i}(m)\right.$ which is the expected output power of the array) with respect to $\mathbf{w}_{i}(m)$ after the $m$ th iteration. The algorithm is "constrained" because the weight vector satisfies the constraint at each iteration, that is $\mathbf{w}_{i}^{H} \mathbf{a}_{i}\left(\theta_{i, g_{\max }}\right)=1$. Rewrite the CLMS algorithm as follows (Xinyu et al., 2008).

$$
\mathbf{w}_{i}(m+1)=\hat{\mathbf{a}}_{i}\left(\mathbf{w}_{i}(m)+\mu g\left(\mathbf{w}_{i}(m)\right)\right)+\frac{\mathbf{a}_{i}\left(\theta_{i, g_{\max }}\right)}{N}
$$

where

$$
\boldsymbol{\beta}_{i}=\mathbf{I}-\frac{\mathbf{a}_{i}\left(\theta_{i, g_{\max }}\right) \mathbf{a}_{i}\left(\theta_{i, g_{\max }}\right)^{H}}{N}
$$

The gradient of $\mathbf{w}_{i}^{H}(m) \mathbf{R}_{x} \mathbf{w}_{i}(m)$ with respect to $\mathbf{w}_{i}(m)$ is given by

$$
g\left(\mathbf{w}_{i}(m)\right) \stackrel{\Delta}{=}=-\frac{\partial}{\partial \mathbf{w}_{i}^{*}}\left(\mathbf{w}_{i}^{H}(m) \mathbf{R}_{\boldsymbol{x}} \mathbf{w}_{i}(m)\right)=-2 \mathbf{R}_{x} \mathbf{w}_{i}(m)
$$

and its computation using this expression requires knowledge of $\mathbf{R}_{\mathbb{x}}$, which normally is not available in practice. For a standard LMS algorithm, an estimate of the gradient at each iteration is made by replacing $\mathbf{R}_{\mathbf{x}}$ by its noise sample $\mathbf{x}_{i}(m+1) \mathbf{x}_{i}(m+1)^{H}$ available at time instant $(m+1)$, leading to

$$
g\left(\mathbf{w}_{i}(m)\right)=-2 \mathbf{x}_{i}(m+1) y_{i}^{*}(m)
$$


The CLMS is a fast convergence algorithm. However, it is drastically sensitive to the mismatch in the direction of arrival. Meanwhile, the weights estimated by the standard algorithm are sensitive to the signal power, requiring a lower step size in the presence of a strong signal for the algorithm to converge, which in turn regarding the decrease of mis-adjustment error, the convergence time is increased (Litva \& Kwok-Yeung, 1996; Haykin, 1996; Xinyu et al., 2008).

Consider the problem of beamforming as to maximize the SINR for a specific link, which is equivalent to minimizing the interference at the receiver of that link. In order to minimize the variance or average power at the output of the beamformer subject to maintaining unity gain at the direction of the desired user signal. Accordingly, we can rewrite the average output power for user $i$ as (Rashid-Farrokhi et al., 1998)

$$
\zeta_{i}=\hat{\mathbf{w}}_{i}^{H} \mathbf{R}_{x} \hat{\mathbf{w}}_{i}
$$

where $\hat{\mathbf{w}}_{i}$ is the optimum weight vector for user $i$ with CLMS algorithm. If the message signals $s_{i}(t)$ are uncorrelated and zero mean, the correlation matrix $\mathbf{R}_{\mathbf{x}}$ is given by

$$
\begin{aligned}
\mathbf{R}_{x x} & =P^{\prime} \mathbf{v}_{i}(\theta) \mathbf{v}_{i}^{H}(\theta)+\sum_{\substack{k=1 \\
k \neq i}}^{M} P^{\prime} \mathbf{v}_{k}(\theta) \mathbf{v}_{k}^{H}(\theta)+\sigma_{n}^{2} \mathbf{I} \\
& =P^{\prime} \mathbf{v}_{i}(\theta) \mathbf{v}_{i}^{H}(\theta)+\boldsymbol{\Phi}_{i}
\end{aligned}
$$

where

$$
\boldsymbol{\Phi}_{i}=P^{\prime} \sum_{\substack{k=1 \\ k \neq i}}^{M} \mathbf{v}_{k}(\theta) \mathbf{v}_{k}^{H}(\theta)+\sigma_{n}^{2} \mathbf{I}
$$

is the correlation matrix of unwanted signals for user $i$ in BS, and $\sigma_{n}^{2}$ is the noise power at the input of each array element. Combining (15)-(17), we obtain the received signal plus interference power as a function of weight vector

$$
\zeta_{i}=P^{\prime}\left(\left|\hat{\mathbf{w}}_{i}^{H} \mathbf{v}_{i}(\theta)\right|^{2}+\sum_{\substack{k=1 \\ k \neq i}}^{M} \hat{\mathbf{w}}_{i}^{H} \mathbf{v}_{k}(\theta) \mathbf{v}_{k}^{H}(\theta) \hat{\mathbf{w}}_{i}\right)+\sigma_{n}^{2} \hat{\mathbf{w}}_{i}^{H} \hat{\mathbf{w}}_{i}
$$

Accordingly, the SINR at BS for user $i$ can be written as follows (Rashid-Farrokhi et al., 1998)

$$
\operatorname{SINR}_{i}(\hat{\mathbf{w}}, \mathbf{v}(\theta))=\frac{P^{\prime}\left|\hat{\mathbf{w}}_{i}^{H} \mathbf{v}_{i}(\theta)\right|^{2}}{P^{\prime} \sum_{\substack{k=1 \\ k \neq i}}^{M}\left|\hat{\mathbf{w}}_{i}^{H} \mathbf{v}_{k}(\theta)\right|^{2}+\sigma_{n}^{2} \hat{\mathbf{w}}_{i}^{H} \hat{\mathbf{w}}_{i}}
$$

It should be mentioned that for the array antenna weight vector elements in the CLMS algorithm and for big $\mu$, will converge after a few iteration (is approximately equal to the number of beamformer weights, i.e., $m=N$ ) (Dosaranian-Moghadam et al., 2010; Dosaranian-Moghadam et al., 2010; Dosaranian-Moghadam et al., 2010; Dosaranian-Moghadam et al., 2011). Moreover it is obvious that, without power control algorithm, the calculated optimal weight vector does not guarantee desirable SINR for the desired user. In simulation section, we will consider perfect power control for increasing SINR of the desired user.

\subsubsection{Conjugate gradient adaptive beamforming}

It is well known that an array of $N$ weights has $N-1$ degree of freedom for adaptive beamforming. This means that with an array of $N$ weights, one can generate $N-1$ pattern nulls and a beam maximum in desired directions. Accordingly, we assume a single-cell wireless system, with $M$ users connected to the base station. Thus, the number of interference signals is $\mathrm{K}=M-1$ that $L$ is the number of paths for each link. To nullify all of these interference signals, one would have to have $\mathrm{K}+1$ weights, which is not practical. So, we focus only on the $L$ paths of the desired user (inter-path interference). Thus, the minimum number of the antenna array weights is $L$ where, typically, $L$ varies from 2 to 6 (Rashid-Farrokhi, 1998). 
In this paper, we use the CGBF algorithm that is used based on orthogonal principle (Mohamed \& Dunham, 1999; Mohamed \& Dunham, 2002; Dosaranian-Moghadam et al., 2010a; Dosaranian-Moghadam et al., 2010b). On this basis, a set of vectors $\mathbf{w}_{k}$ is to be selected such that they are $\mathbf{A}$-orthogonal, i.e., $\left\{\mathbf{A w}_{k}, \mathbf{A w}_{k^{\prime}}\right\rangle=0$ for $k \neq k^{\prime}$. In this algorithm, at each base station, an antenna array of $S$ sensors and $N$ weights is employed, where $S=2 N-1$ for $n=-(N-1), \ldots,-1,0,1, \ldots,+(N-1)$, to receive signals from all users. Note that in CGBF adaptation algorithm, unlike other adaptation algorithms, for example, the CLMS algorithm, the number of weights is less than the number of sensors. Accordingly, the optimum weights of $j$ th branch of the RAKE receiver for the CGBF algorithm at time $m$ are obtained by minimizing (Mohamed \& Dunham, 1999; Mohamed \& Dunham, 2002; Dosaranian-Moghadam et al., 2010; Dosaranian-Moghadam et al., 2010)

$$
\left\|\mathbf{\Psi}_{i}^{(j)}(m)\right\|^{2}=\mathbf{\Psi}_{i}^{H(j)}(m) \mathbf{\Psi}_{i}^{(j)}(m)
$$

where

$$
\boldsymbol{\Psi}_{i}^{(j)}(m)=\mathbf{A}_{i} \mathbf{w}_{i}^{(j)}(m)-\mathbf{y}_{i}^{(j)}
$$

and using (6),

$$
\mathbf{A}_{i}=\left[\begin{array}{ccc}
x_{-(N-1)} & \ldots & x_{0} \\
\cdot & \cdot & \cdot \\
x_{0} & \ldots & x_{+(N-1)}
\end{array}\right]
$$

is the $N \times N$ signal matrix. Also,

and

$$
\mathbf{y}_{i}^{(j)}=\left[\begin{array}{lllll}
e^{-j(N-1) \theta / 2} & \ldots 1 \ldots & e^{+j(N-1) \theta / 2}
\end{array}\right]^{T}
$$

$$
\mathbf{w}_{i}^{(j)}(m)=\left[w_{i, 0}^{(j)}(m) w_{i, 1}^{(j)}(m) \quad \ldots \quad w_{i, N-1}^{(j)}(m)\right]^{T}
$$

are the excitation and weight vectors $(N \times 1)$ for desired user in the $j$ th path, respectively.

It should be mentioned that the CGBF algorithm has two main characteristics (Mohamed \& Dunham, 2002):

1. This algorithm can produce a solution of the matrix equation very efficiently and converge in a finite number of iterations (the number of beamformer weights).

2. Convergence is guaranteed for any possible condition of the signal matrix, according to (22).

According to the algorithm of CGBF, the updated value of the weight vector for desired user in the $j$ th path at time $m+1$ is computed by using the simple recursive relation (Mohamed \& Dunham, 2002; Mohamed \& Dunham, 2002):

$$
\mathbf{w}_{i}^{(j)}(m+1)=\mathbf{w}_{i}^{(j)}(m)+\kappa_{i}^{(j)}(m) \boldsymbol{\beta}_{i}^{(j)}(m)
$$

where

$$
\begin{aligned}
& \kappa_{i}^{(j)}(m)=\left\|\mathbf{A}_{i}^{H} \boldsymbol{\Psi}_{i}^{(j)}(m)\right\|^{2} /\left\|\mathbf{A}_{i} \boldsymbol{\beta}_{i}^{(j)}(m)\right\|^{2} \\
& \boldsymbol{\Psi}_{i}^{(j)}(m+1)=\boldsymbol{\Psi}_{i}^{(j)}(m)+\boldsymbol{\kappa}_{i}^{(j)}(m) \boldsymbol{\beta}_{i}^{(j)}(m) \\
& \boldsymbol{\beta}_{i}^{(j)}(0)=-\mathbf{A}_{i}^{H} \boldsymbol{\Psi}_{i}^{(j)}(0) \\
& \boldsymbol{\beta}_{i}^{(j)}(m+1)=\mathbf{A}_{i}^{H} \boldsymbol{\Psi}_{i}^{(j)}(m+1)+\eta_{i}^{(j)}(m) \boldsymbol{\beta}_{i}^{(j)}(m) \\
& \eta_{i}^{(j)}(m)=\left\|\mathbf{A}_{i}^{H} \boldsymbol{\Psi}_{i}^{(j)}(m+1)\right\|^{2} /\left\|\mathbf{A}_{i}^{H} \boldsymbol{\Psi}_{i}^{(j)}(m)\right\|^{2}
\end{aligned}
$$


Accordingly, we can rewrite (18) for the CGBF algorithm as follows.

$$
\zeta_{i}^{j}=P^{\prime}\left(\left|\hat{\mathbf{w}}_{i}^{H(j)} \mathbf{v}_{i}^{(j)}(\theta)\right|^{2}+\sum_{\substack{k=1 \\ k \neq i}}^{M} g_{i}^{(j)}(\theta) \hat{\mathbf{w}}_{i}^{H} \mathbf{v}_{k}(\theta) \mathbf{v}_{k}^{H}(\theta) \hat{\mathbf{w}}_{i}\right)+\sigma_{n}^{2} \hat{\mathbf{w}}_{i}^{H} \hat{\mathbf{w}}_{i}
$$

where

$$
\begin{aligned}
& \mathbf{v}_{i}^{(j)}(\theta)=\sum_{l=1}^{L_{i}} \sqrt{\alpha_{i, l}} \mathbf{a}_{i}\left(\theta_{i, l}\right) g_{i}^{(j)}(\theta) \\
& =\underbrace{\sqrt{\alpha_{i, 0}} \mathbf{a}_{i}\left(\theta_{i, 0}\right)+\ldots+\sqrt{\alpha_{i, j-1}} \mathbf{a}_{i}\left(\theta_{i, j-1}\right)}_{\text {IPI }=\mathbf{0}}+\sqrt{\alpha_{i, j}} \mathbf{a}_{i}\left(\theta_{i, j}\right)+\underbrace{\sqrt{\alpha_{i, j+1}} \mathbf{a}_{i}\left(\theta_{i, j+1}\right) \ldots+\sqrt{\alpha_{i, L_{i}}} \mathbf{a}_{i}\left(\theta_{i, L_{i}}\right)}_{\mathbf{I P I}=\mathbf{0}} \\
& =\sqrt{\alpha_{i, j}} \mathbf{a}_{i}\left(\theta_{i, j}\right)
\end{aligned}
$$

and

$$
g_{i}^{(j)}(\theta)=\left[e^{-j(N-1) \theta / 2} \ldots 1 \ldots e^{+j(N-1) \theta / 2}\right] \times \mathbf{w}_{i}^{(j)}
$$

$\mathrm{www}_{\text {is }}$ is the magnitude response of the $j$ th beamformer for user $i$ toward the direction of arrival $\theta$ and $\mathbf{w}_{i}^{(j)}$ is the $j$ th beamformer's weight vector for user $i$.

Accordingly, the desired user resolvable directions are fed to the conjugate gradient beamformer to cancel out the inter-path interference (IPI) from other directions. Also, the output signals from the beamformer with conventional maximal ratio combining (MRC) are combined and then are fed into the decision circuit of the desired user. Hence, by the CGBE algorithm, desired users' signal in an arbitrary path is passed and the IPI is canceled in other paths in each RAKE finger, while the IPI is not removed in the CLMS algorithm.

\subsection{The Switched-Beam Technique}

One simple alternative to the fully adaptive antenna is the switched-beam architecture in which the best beam is chosen from a number of fixed steered beams. Switched-beam systems are technologically the simplest and can be implemented by using a number of fixed, independent, or directional antennas (Gotsis et al., 2009). We list the conditions of the SB technique for this paper as follows (Dosaranian-Moghadam et al., 2010).

1. According to Fig. 3, beams coverage angle is $30^{\circ}$ and overlap between consecutive beams is. $20^{\circ}$ Thus each base station has 36 beams.

2. According to Fig. 4, each user can use one beam for each of its path to communicate with a base station at any time.

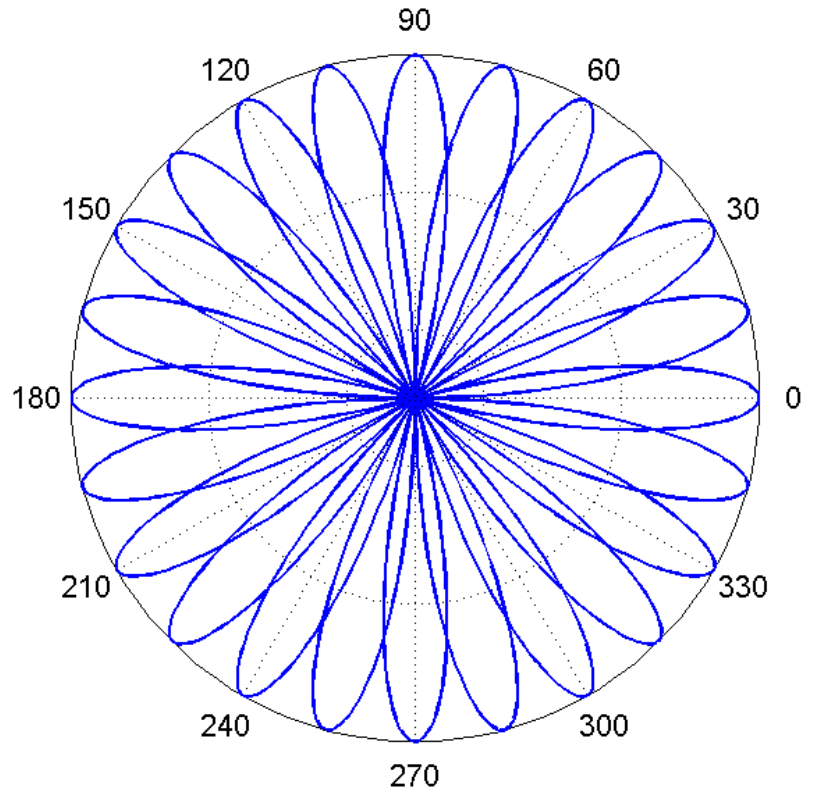

Fig.3. 36 beams in each base station with switched-beam technique 


\subsubsection{Equal sectoring method}

One simple method used to sectorize a cell is equal sectoring; in which all sectors have the same coverage angle. In this paper, we assume 12 sectors for each base station with sector angle $30^{\circ}$ for the ES method (Dosaranian-Moghadam et al., 2010).

\subsection{Power control error}

When imperfections in power control are considered, multipath fading is not perfectly compensated. As a result, the power received from a mobile will not be constant at the base station to which the mobile is connected. Accordingly, we can rewrite (1) as follows (Mohamed \& Dunham, 2002; Romero-Jerez et al., 2004)

$$
\mathbf{x}(t)=\sum_{i=1}^{M} \sqrt{P^{\prime} \lambda_{i}} s_{i}\left(t-\tau_{i}\right) \mathbf{v}_{i}(\theta)+\mathbf{n}(t)
$$

where the variable $\lambda_{i}$ is PCE for user $i$, which is assumed to follow a log-normal distribution and thus can be written as $\lambda_{i}=10^{v_{i} / 10}$ where $v_{i}$ is a Gaussian random variable with mean 0 and variance $\sigma_{v}^{2}$ for all users (Carrasco \& Femenias, 2008).

On the other hand, $\mathrm{E}\left[\lambda_{i}\right]$ for all users can be written as follows (Romero-Jerez et al., 2004).

$$
\mathrm{E}\left[\lambda_{i}\right]=e^{\beta^{2} \sigma_{v}^{2} / 2}
$$

where. $\beta=\ln (10) / 10$ Accordingly, we can rewrite the SINR in (25) for the CLMS algorithm as follows.

$$
\operatorname{SINR}_{i}(\hat{\mathbf{w}}, \mathbf{v}(\theta))=\frac{P^{\prime} e^{\beta v_{i, q}}\left|\hat{\mathbf{w}}_{i}^{H} \mathbf{v}_{i}(\theta)\right|^{2}}{P^{\prime} e^{\beta^{2} \sigma_{v}^{2} / 2} \sum_{\substack{k=1 \\ k \neq i}}^{M}\left|\hat{\mathbf{w}}_{i}^{H} \mathbf{v}_{k}(\theta)\right|^{2}+\sigma_{n}^{2} \hat{\mathbf{w}}_{i}^{H} \hat{\mathbf{w}}_{i}}
$$

Fig.4. Select of beam for two users in two paths with switched-beam technique.

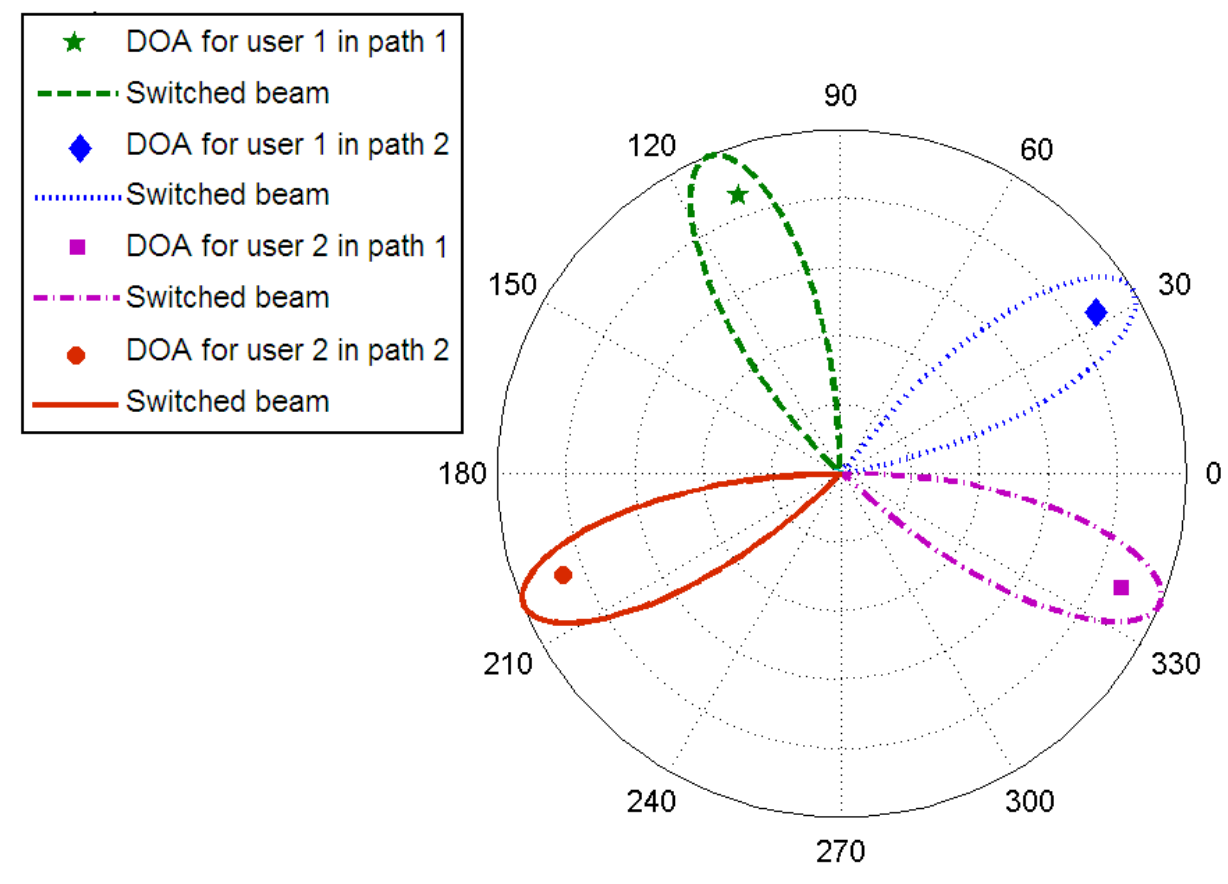

\subsection{Simulation results}

In this section, we investigate the effect of the PCE on the SINR performance of wireless cellular systems in a 2D urban environment. Accordingly, we chose part of Two-dimensional map of the University of Toronto campus area in Canada as shown in Fig. 5 (Dosaranian-Moghadam et al., 2009; Dosaranian-Moghadam et al., 2012). On the other hand, we assume beside main user, $M-1$ interference users in a stable positioning situation are also sending information to base station equipped to an array antenna in reverse link. For this and also according to the description in Section 2, sending information by all users to base station is performed 
by dramatizer USPS. Also, we assume $L_{i}=L=4$ resolvable propagation paths for all users for all links. In addition, we assume the resolution, path loss parameter, and variance of the log-normal shadow fading in USPS $R=1, L_{p}=0.005 \mathrm{~dB} / \mathrm{m}$, and $4 \mathrm{~dB}$ respectively. We also assume that the variance of the noise $\left(\sigma_{n}^{2}\right)$ for every element is assumed to be equal to 0.01 in all our simulations.

In Fig. 6, the SINR of desired user in reverse link for the case of PPC versus the number of interference users $(M-1)$ is compared. In this simulation, we use the SB, CGBF, CLMS, and ES methods for, $N=10,15 d=0.5 \lambda, 0.75 \lambda$ path loss parameter $L_{P}=0.005 \mathrm{~dB} / \mathrm{m}$ , and $\mu=0.005$. This figure shows that the SINR for desired user with SB technique is higher than that with the other methods. On the other hand, the figure shows the SINR for desired user with CGBF algorithm is higher than that with CLMS algorithm, because in CGBF algorithm the IPI is removed whereas in CLMS algorithm it is not canceled. Also, we observe that the SINR in the ES method is lower than that in the other methods, because in this method the interference is higher than that in CLMS and CGBF algorithms and SB technique. On the other hand, it can be seen that with $\mathrm{N}=15$ the beamforming method has better performance in comparison with the case $\mathrm{N}=10$. In addition, we can see that the SINR of desired user for $d=0.5 \lambda$ is better than that for $d=0.75 \lambda$ (see (Dosaranian-Moghadam et al., 2009) for more details).

Fig. 7 shows the influence of path-loss parameter $\left(L_{p}\right)$ in USPS on the SINR of desired user for the CGBF algorithm for the PPC anD N=10. The figure shows, as expected, a decrease in the path-loss parameter entails an increase in the interference and desired signal levels and, therefore, an improvement in system performance using antenna arrays in the base station. For example, at a SINR of $12 \mathrm{~dB}$ and for the CGBF algorithm, the number of interference users is, respectively, 19,25 , and 30 users for $L_{p}=0.03 \mathrm{~dB} / \mathrm{m}$ $L_{p}=0.01 \mathrm{~dB} / \mathrm{m}$ and $L_{p}=0.005 \mathrm{~dB} / \mathrm{m}$

Results for the SINR versus the number of interference users $(M-1)$ and different values of $\sigma_{v}^{2}$ for the CGBF algorithm and $\mathrm{N}=10$ are provided in Fig. 8. It can be seen that the SINR for $\sigma_{v}^{2}=0 \mathrm{~dB}$ (perfect power control) is higher than that for the other cases. Also, it is seen that if $\sigma_{v}^{2}$ increases from 4 to $8 \mathrm{~dB}$, the number of active users decreases by approximately $42 \%$ for a SINR of $12 \mathrm{~dB}$, whereas if $\sigma_{v}^{2}$ increases from 2 to $4 \mathrm{~dB}$, the capacity decreases by approximately $40 \%$.

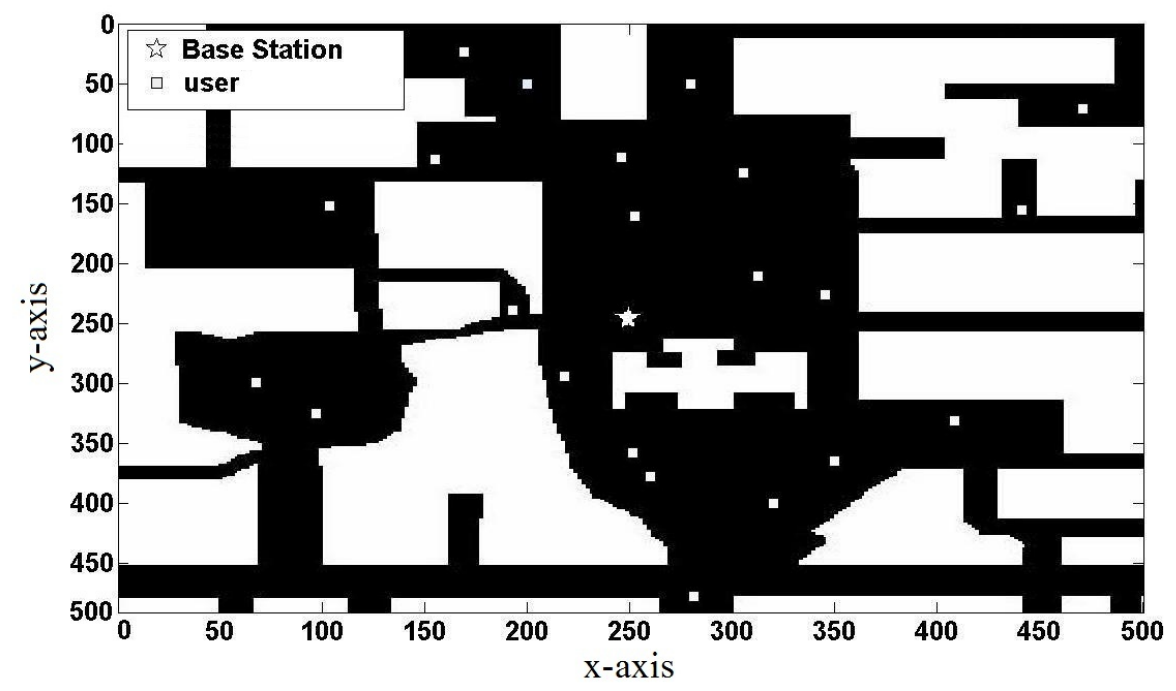

Fig.5. Two-dimensional map of the University of Toronto campus area and placing $M$ users and base station

The influence of the variance of PCE on the system capacity is illustrated in Fig. 9 for SB, CGBF, and CLMS methods. As expected, the number of active users decreases as the variance of PCE increases. For example, for CGBF technique, the number of users allowed in the cell is 20 users for $2 \mathrm{~dB}$, while the number of active users is 7 users for $\sigma_{v}^{2}=8 \mathrm{~dB}$. Also, it can be seen that the number of users allowed in the system for SB technique is higher than that for CGBF and CLMS algorithms. 
Fig.6. The SINR of desired user in reverse link versus the number of interference users (M-1) for SB, CGBF, CLMS, and ES methods

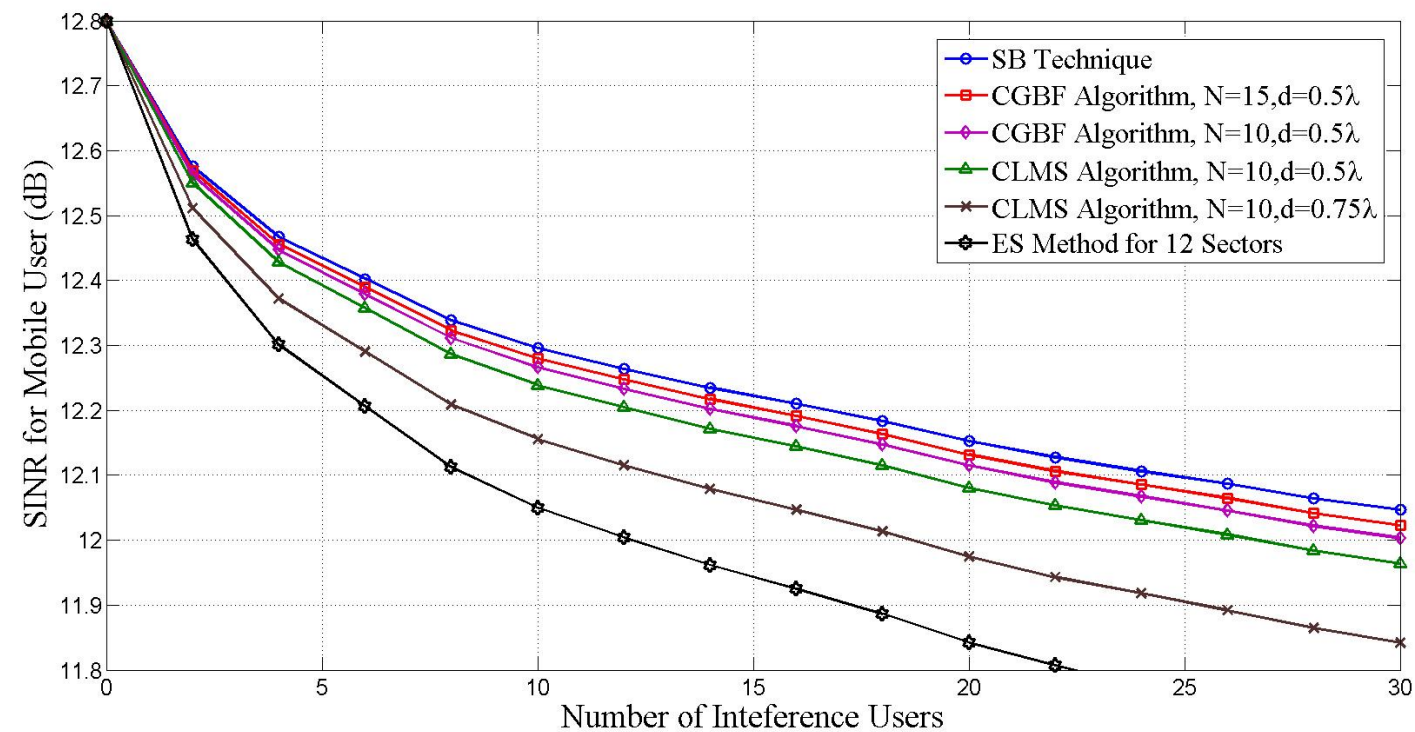

In addition, the number of active users for the CLMS algorithm is lower than that for CGBF algorithm. For example, for $\sigma_{v}^{2}=4 \mathrm{~dB}$ , the number of users allowed in the cell is 22 users for SB technique, while for CGBF and CLMS algorithms, the number of users is 12 users and 10 users, respectively.

Fig. 7. Influence of path-loss parameter on the SINR of desired user for the CGBF algorithm for the case of PPC

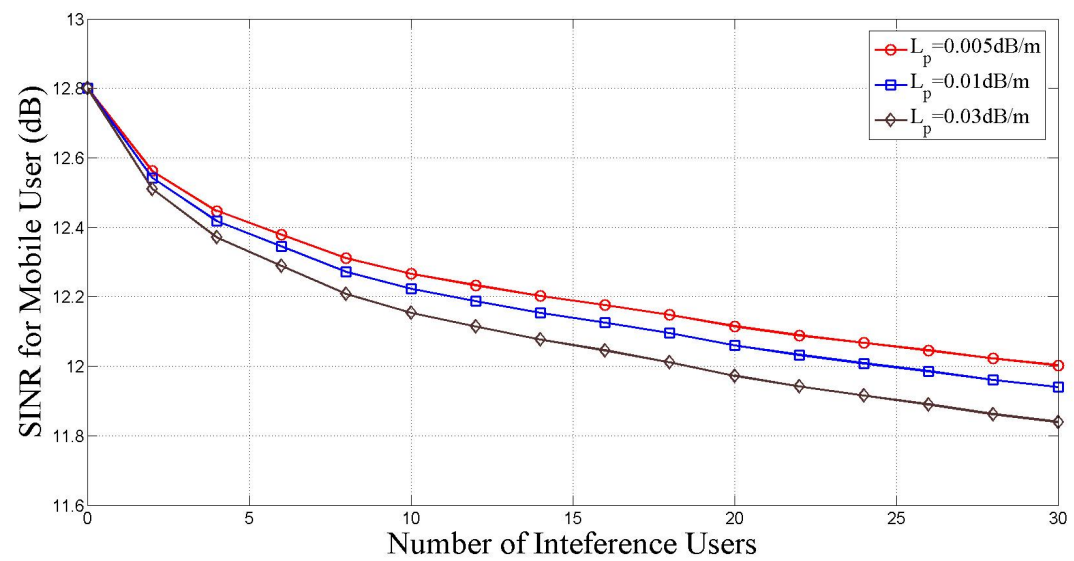

Fig.8. The SINR of desired user for the CGBF algorithm for different values of $\sigma_{v}^{2}$

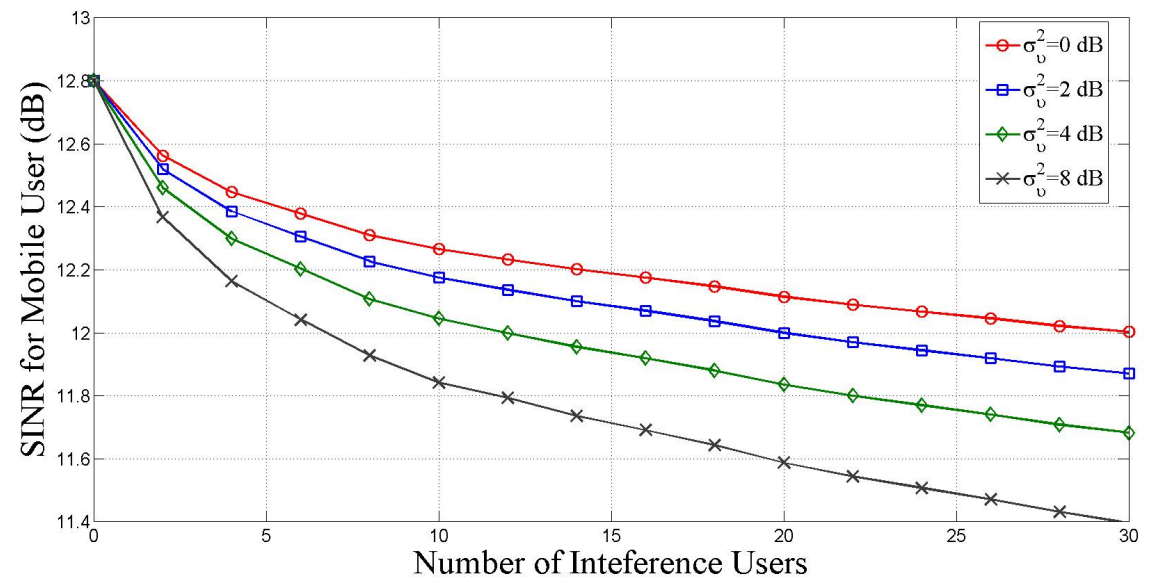


Fig.9. The SINR of desired user for different values of $\sigma_{v}^{2}$

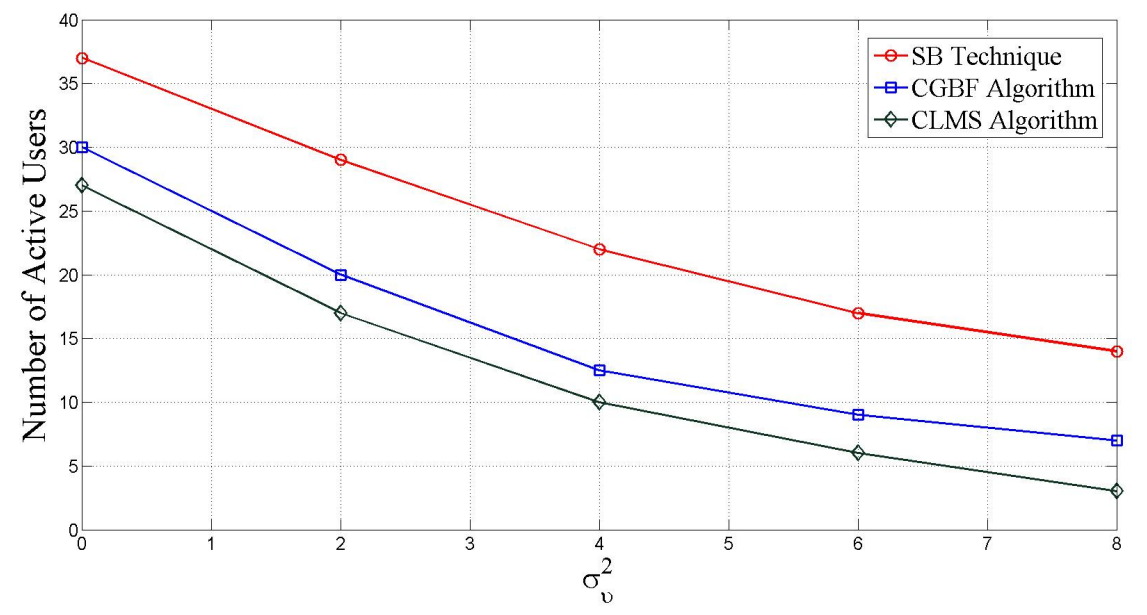

\section{Conclusions}

In this paper, we studied joint adaptive beamforming algorithm and perfect power control in a 2D urban environment in reverse link. The performance of the system is observed for uniformly spaced linear array by varying the inter-element spacing between the array elements for perfect power control and power control error. Accordingly, it has been observed that the SINR of desired user with the CGBF algorithm is higher than that with the CLMS algorithm, because in the CGBF algorithm, the IPI is removed whereas in CLMS algorithm it is not canceled. It has also been shown that the SINR of desired user in the SB technique is lower than that in the CGBF algorithm. On the other hand, it has been shown that that the SINR for $d=0.5 \lambda$ is better than that for the other cases. It has also been observed that decreasing the path loss parameter $\left(L_{p}\right)$ improves the SINR of desired user. Also our simulations show that the variations in power level due to PCE have a detrimental effect on system performance.

\section{References}

1 Abdel-Samad, Davidson TN and Gershman AB (2006) Robust transmit eigen beamforming based on imperfect channel state information. IEEE Trans. Signal Processing. 54(5), 1596-1609.

2- Abrardo (2003) Noncoherent MLSE in DS-CDMA wireless systems with antenna arrays. IEEE Trans. Vehicular Technol. 52(6), $1435-1446$.

3- Abrardo and Sennati D (2000) On the analytical evaluation of closed-loop power-control error statistics in DS-CDMA cellular systems. IEEE Trans. Vehicular Technol. 49(6), 2071-2080.

4- Akhlaghi, Mirsalehi MM and Zamiri H (2004) Radio signal simulator for urban areas. Iran. Conf. Elect. Engg.(ICEE2004), Mashhad, Iran.

5- Cai Y and de Lamare RC (2009) Space-time adaptive MMSE multiuser decision feedback detectors with multiple-feedback interference cancellation for CDMA systems. IEEE Trans. Vehicular Technol. 58(8), 4129-4140.

6• Carrasco L and Femenias G (2008) Reverse link performance of a DS-CDMA system with both fast and slow power controlled users. IEEE Trans. Wireless Commun. 7(4), 1255-1263.

7• Chang J, Tassiulas L and Rashid-Farrokhi F (2003) Joint transmitter receiver diversity for efficient space division multi-access. IEEE Trans. Wireless Commun. 1(1), 6-27.

8- Dosaranian-Moghadam M and Bakhshi H (2012) Tracking mobile user through adaptive beamforming for wireless cellular networks in a 2D Urban environment. Indi. J. Sci. \& Technol. 5(4), 2569-2577.

9• Dosaranian-Moghadam M, Bakhshi H and Dadashzadeh G (2010) Interference management for DS-CDMA systems through closed-loop power control, base station assignment, and beamforming. J. Wireless Sensor Network. 2(6), 472-482.

10 Dosaranian-Moghadam M, Bakhshi H and Dadashzadeh G (2010) Joint centralized power control and cell sectoring for interference management in CDMA cellular systems in a 2D Urban environment. J. Wireless Sensor Network. 2(8), 599-605. 
11 Dosaranian-Moghadam M, Bakhshi H and Dadashzadeh G (2010) Joint closed-loop power control and adaptive beamforming for wireless networks with antenna arrays in a 2D urban environment. $J$ Wireless Sensor Network. 2(11), 869-878.

12. Dosaranian-Moghadam M, Bakhshi $\mathrm{H}$ and Dadashzadeh G (2010b) Interference management for DS-CDMA receiver through base station assignment in multipath fading channels. Proc. 2010 IEEE Intl. Conf. Wireless Commun., Networking \& Info. Security, Beijing, China, 6, 257-263.

13. Dosaranian-Moghadam M, Bakhshi H and Dadashzadeh G (2011) DS-CDMA cellular systems performance with base station assignment, power control error and beamforming over multipath fading. Intl. J. Comput. Networks \& Commun. 3(1), 185-202.

14- Dosaranian-Moghadam M, Bakhshi H, Dadashzadeh G and Godarzvand-Chegini M (2010) Joint closed-loop power control and constrained LMS algorithm for DS-CDMA receiver in multipath fading channels. Proc. 2010 IEEE Global Mobile Congress, Shanghai, China. 18-19(10), 1-8.

15- Dosaranian-Moghadam M, Bakhshi H, Dadashzadeh G and Godarzvand-Chegini M (2010a) Joint base station assignment, Power control error, and adaptive beamforming for DS-CDMA Cellular systems in multipath fading channels. Proc. 2010 IEEE Global Mobile Congress, Shanghai, China. 18-19(11), 1-7.

16• Dosaranian-Moghadam M, Bakhshi H, Dadashzadeh G and Rahmati P (2009) Adaptive beamforming method based on constrained LMS algorithm for tracking mobile user. Proc. 2009 IEEE Global Mobile Congress, Shanghai, China. 10, 1-6.

17• Dosaranian-Moghadam M, H. Bakhshi, and G. Dadashzadeh (2010) Joint constrained LMS algorithm and base station assignment for DS-CDMA receiver in multipath fading channels. Proc. 2010 IEEE Wireless Commun., Networking \& Mobile Comput., Chengdu, China. 23-25(9), 1-7.

18- Gotsis KA, Siakavara K and Sahalos JN On the direction of arrival (DoA) Estimation for a switched-beam antenna system using neural networks. IEEE Trans. Antennas \& Propagation. 57(5), 1399-1411.

19• Haykin S (1996) Adaptive filter theory. $3^{\text {th }}$ ed., New Jersey. Prentice Hall.

20- Keyi El and Champagne B (2010) Adaptive linearly constrained minimum variance beamforming for multiuser cooperative relaying using kalman filter. IEEE Trans. Wireless Commun. 9(2), 641-6510.

21 Kumatani K, McDonough J, Rauch B, Klakow D, Garner PN and Li W (2009) Beamforming with a maximum negentropy criterion.IEEE Trans. Audio, Speech \& Language Processing. 17(5), 994-1008.

22 - Lawrence DE (2010) Low probability of intercept antenna array beamforming. IEEE Trans. Antennas \& Propagation. 58(9), 2858-2865.

23• Litva J and Kwok-Yeung T (1996) Digital beamforming in wireless Communication. Artech House.

24• Lorenz RG and Boyd SP (2005) Robust minimum variance beamforming. IEEE Trans. Signal Processing. 53(5), 1684-1696.

25. Mohamed NA and Dunham JG (1999) Adaptive beamforming for DS-CDMA using conjugate gradient algorithm in a multipath fading channel. Proc. 1999 IEEE Emerging Technol. Sym., Dallas, TX. 12-13(4), 859-863.

26. Mohamed NA and Dunham JG (2002) A low-complexity combined antenna array and interference cancellation DS-CDMA receiver in multipath fading channels. IEEE J. Selected Areas in Commun. 20(2), 248-256.

27• Nai SE, Ser W, Yu ZL and Rahardja S (2009) A robust adaptive beamforming framework with beampattern shaping constraints. IEEE Trans. Antennas \& Propagation. 57(7), 2198-2203.

28- Park SH, Lee H, Lee SR and Lee I (2009) A new beamforming structure based on transmit-MRC for closed-loop MIMO systems. IEEE Trans. Commun. 57(6), 1847-1856.

29- Qian L and Gajic Z (2006) Variance minimization stochastic power control in CDMA system. IEEE Trans. Wireless Commun. 5(1), 193-202.

30• Rashid-Farrokhi F, Ray-Liu KJ and Tassiulas L (1998) Transmit beamforming and power control for cellular systems. IEEE J. Selected Areas in Commun. 16(8), 1437-1450.

31 Rashid-Farrokhi F, Tassiulas L and Ray-Liu KJ (1998) Joint optimal power control and beamforming in wireless networks using antenna arrays. IEEE Trans. Commun. 46(10), 1313-1324.

32. Rintamaki M, Koivo H and Hartimo I (2004) Adaptive closed-loop power control algorithms for CDMA cellular communication systems. IEEE Trans. Vehicular Technol. 53(6), 1756-1768. 
33- Romero-Jerez JM, Tellez-Labao C and Diaz-Estrella A (2004) Effect of power control imperfections on the reverse link of cellular CDMA networks under multipath fading. IEEE Trans. Vehicular Technol. 53(1), 61-71.

34 Shakir MZ and Durrani TS (2007) Narrowband beamforming algorithm for smart antennas. Int. Bhurban Conf. Appl. Sci. \& Technol. 1, 49-54.

35- Wang J and Payaro M (2010) On the robustness of transmit beamforming. IEEE Trans. Signal Processing. 58(11), 5933-5938.

36- Wang J and Yu A (2001) Open-loop power control error in cellular CDMA overlay systems. IEEE J. Selected Areas in Commun. 19(7), 1246-1254.

37• Wang JT (2009) Admission control with distributed joint diversity and power control for wireless networks. IEEE Trans. Vehicular Technol. 58(1), 409-419.

38- Xinyu S, Xiaohua L and Jianjiang Z (2008) Robust adaptive beamforming based on maximum likelihood estimation. Int. Conf. Microwave \& Millimeter Wave Technol. 3(4), 1137-1140.

39• Yang LL and Fang W (2009) Performance of distributed-antenna DS-CDMA systems over composite lognormal shadowing and Nakagami-m-fading channels. IEEE Trans. Vehicular Technol. 58(6), 2872-2883.

40- Ye W and Haimovich AM (2000) Performance of cellular CDMA with cell site antenna array, Rayleigh fading, and power control error. IEEE Trans. Commun. 48(7), 1151-1159.

41 Zhang R, Chai CC and Liang Y (2009) Joint beamforming and power control for multiantenna relay broadcast channel with QoS constraints. IEEE Transact. on Signal Processing. 57(2), 726-737. 